Macedonian Pharmaceutical Bulletin, 66 (Suppl 1) 133 - 134 (2020)

Online ISSN 1857 - 8969

UDC: 615.453.6.014.2

DOI: 10.33320/maced.pharm.bull.2020.66.03.066

Short communication

\title{
Optimization of tablet compressing process using experimental design approach
}

\author{
Katerina Tnokovska*, Krume Toshev, Bojana Trifunovska Vulovska, Packa Antovska, \\ Sonja Ugarkovic
}

Research \& Development, Alkaloid AD, Blvd. Aleksandar Makedonski 12, 1000 Skopje, R. N. Macedonia

\section{Introduction}

Identification of critical process parameters (CPPs) that can have an effect on product Critical Quality attributes (CQAs) and determining the functional relationships that link process parameters to product CQAs is critical step in understanding the process and process control (ICH quideline Q8).

According to the initial risk assessment, performed during formulation/ process development, regarding the link between Critical Quality Attributes and Critical Process Parameters, related to the equipment connected with the technological process of the product containing BCS III active substance, tableting speed and compression force have been assessed as critical process parameters in tablet compressing process (Tho and Bauer-Brandl, 2011).

The aim of this study was to optimize the tablet compression process and to predict the ability of the process to produce product with quality attributes which are within a predetermined range in order to ensure final product quality.

\section{Materials and methods}

In order to optimize CPPs in tablet compression process and assess the impact on average mass, friability, thickness, hardness, dissolution and disintegration as CQAs, Quality by Design (QbD) approach has been applied. The goal is to approximate the response by a mathematical model for the purpose of optimization and finding a region of operability (normal operating range).

The robustness/optimization study has been performed on one industrial batch of the product in the Production Department. $2^{2}$ full factorial design with two replicates in the center point has been applied with varying two factors: tableting speed (tbl/hour) and main compression force $(\mathrm{kN})$, adjusted to obtain low and high levels of tablet hardness on the upper and lower limit of the target range, in order to provide flexibility in the process while still producing product which meets relevant quality criteria.

Seven experiments were generated, performed and the results for each response were inserted and evaluation of raw data and model interpretation were performed using MODDE Go ${ }^{\circledR}$ statistical software.

Appropriate statistical analysis has been performed regarding the optimization/robustness results in order to estimate the influence of changes in the process parameters on the physico-chemical properties of the tablets (Eriksson et al., 2008).

Additionally, the power failure case on the compression process of the product has been tested

\footnotetext{
*ktnokovska@alkaloid.com.mk
} 
with process control analysis of physico-chemical parameters. The results of these experiments has been used to optimize the tablet compression process and predict the process's ability to produce product within acceptance criteria.

\section{Results and discussion}

Main effects of independent variables on response variables have been evaluated. Tablet hardness increases with increase in the compression force values, due to higher compression force applied. As the compression force increases, thickness progressively decreases due to tightening of the tablet core as result of higher applied compression pressure. Tablets compressed under higher compression forces have slightly lower friability values due to higher tablet hardness. Hardness, thickness and friability are not affected from increasing tablet compression speed from low to high level. Disintegration time of tablets is not generally affected from increasing tablet compression force and tablet compression speed from low to high level. Dissolution of the API is slightly affected due to increased compression force, but the results are within predetermined limit. There is no significant change in mass variation due to change in compression forces applied and compression speed from low to high level. The variation in average mass is within in-process control limits.

Correlation plot demonstrates and confirms the main correlations of factors with the responses, therefore it can be confirmed that compression force is positively correlated to tablet hardness. Negative correlation is observed between the compression force and thickness and compression force and friability and hardness and dissolution.

From the obtained Sweet Spot Plot for the areas where the responses are within specified ranges, we can observe that all responses for concerned dependent variables hardness, thickness, friability, mass variation, disintegration and dissolution are within selected range. Varying independent variables, tablet machine speed from low level (40 $000 \mathrm{tbl} / \mathrm{h})$ to high level $(60000 \mathrm{tbl} / \mathrm{h})$ and compression force from low level $(15 \mathrm{kN})$ to high level $(25 \mathrm{kN})$ provides results for concerned dependent variables within desired range.

The obtained results for all parameters for the power failure case are well within the predetermined acceptance criteria. Dissolution profile in the specification media $\mathrm{pH} 6.8$ has been performed for all cases of the optimization process including for the power failure case and shows similar dissolution profiles for all cases of the tablet compression process.

\section{Conclusion}

Evaluation of tablet compression process by factorial design showed that the tested factors within the tested range have no significant influence on the CQAs of the final product. Each combination of the tested parameters within the tested range would produce product with acceptable quality. Robustness/optimization study of tablet compressing process has been successfully performed and defines the range of baseline compressing parameters which provide reproducibility of the results for quality attributes of the tablet cores during tablet compressing process.

\section{References}

Eriksson, L., Johansson, E., Kettaneh-Wold, N., 2008. Design of Experiments, Principles and Applications. Umetrics Academy 53-101, 317-323.

ICH guideline Q8 (R2) on pharmaceutical development. EMA/CHMP/ICH/167068/2004.

Tho, I., Bauer-Brandl, A., 2011. Quality by design (QbD) approaches for the compression step of tableting. Expert Opinion on Drug Delivery 8(12), 1631-1644.

Maced. Pharm. Bull. 66 (Suppl 1) 133 - 134 (2020) 